\title{
A mosaic maternal splice donor mutation in the EHMT1 gene leads to aberrant transcripts and to Kleefstra syndrome in the offspring
}

\author{
Andreas Rump ${ }^{1}$, Laura Hildebrand ${ }^{1}$, Andreas Tzschach ${ }^{2,3}$, Reinhard Ullmann ${ }^{3}$, Evelin Schrock ${ }^{1}$ and \\ Diana Mitter ${ }^{\star, 4}$
}

The euchromatic histone-lysine $\mathrm{N}$-methyltransferase 1 (EHMT1) gene was examined in a 3-year-old boy with characteristic clinical features of Kleefstra syndrome. Sequencing of all 27 EHMT1 exons revealed a novel mutation, NM_024757.4:c.2712 + 1G $>$ A, which affects the splice donor of intron 18. Whereas the index patient is heterozygous for that mutation, his phenotypically normal mother shows tissue-specific mosaicism. Sequencing of EHMT1 RT-PCR products revealed two aberrant transcript variants: in one variant, exon 18 was skipped; in the other, a near-by GT motif was used as splice donor and intronic sequence was inserted between exons 18 and 19. Both transcript variants were found in the patient and his mother. The latter had lower amounts of these transcripts consistent with mosaic status. This is the first description of an EHMT1 point mutation being inherited from a parent with verified mosaicism. The constitutive c.2712 $1 \mathrm{G}>\mathrm{A}$ splice site mutation in EHMT1 is fully pathogenic, and the transcript variants produced do not attenuate the severity of the disease. European Journal of Human Genetics (2013) 21, 887-890; doi:10.1038/ejhg.2012.267; published online 12 December 2012

Keywords: Kleefstra syndrome; EHMT1; splice donor mutation; mosaicism

\section{INTRODUCTION}

Kleefstra syndrome (OMIM 610253) ${ }^{1}$ is characterized by moderateto-severe intellectual disability, childhood hypotonia, and distinctive facial features. Additional clinical findings include heart defects, renal/ urological defects, genital defects in males, autistic-like behavior in childhood, and extreme apathy or catatonic-like features after puberty. ${ }^{2}$ The genetic causes of Kleefstra syndrome are heterozygous microdeletions in 9q34.3 and EHMT1 (euchromatic histone-lysine $\mathrm{N}$-methyltransferase 1) point mutations, both leading to loss of function of the affected EHMT1 allele. ${ }^{1,3}$ Parent-to-child transmission of Kleefstra syndrome appears to be very rare and was described only for three cases with $9 \mathrm{q} 34.3$ deletion ${ }^{4,5}$ and one case with a balanced translocation. ${ }^{5}$ All EHMT1 point mutations described so far have appeared de novo in the patients. ${ }^{6}$

Here, we report on a patient with characteristic features of Kleefstra syndrome carrying a novel splice-site mutation in EHMT1. The mutation was inherited from the mother who showed tissue-specific mosaicism.

\section{MATERIAL AND METHODS}

Patient

This boy (Figure 1) was the first child born to caucasion non-consanguineous parents, a 19-year-old woman (Figure 1) and a 22-year-old man. No fetal abnormalities were recorded during pregnancy. The boy was born spontaneously after 36 weeks of pregnancy with a birth weight of $2370 \mathrm{~g}(-0.9 \mathrm{SD})$, length $43 \mathrm{~cm}(-2.4 \mathrm{SD})$, and head circumference $31 \mathrm{~cm}(-1.8 \mathrm{SD})$. At birth, a neonatal tooth was noted, which fits into the phenotypic spectrum observed in patients with Kleefstra syndrome. ${ }^{7}$ In addition, the boy showed apnoeic episodes with bradycardia, hyperbilirubinemia, anemia, and neutropenia. Echocardiography was normal. At the age of 1 month, bilateral incarcerated inguinal hernia was corrected. Early development was complicated by failure to thrive and recurrent infections.

On examination at the age of 3 years 10 months, his length was $103 \mathrm{~cm}$ (mean), weight $18 \mathrm{~kg}(1.1 \mathrm{SD})$, and head circumference $47 \mathrm{~cm}(-2.9 \mathrm{SD})$. He showed brachymicrocephaly, characteristic facial dysmorphism (Figure 1), and tapering fingers. He presented with muscular hypotonia. His motor and speech development were delayed. He learned to sit at the age of 12 months and to walk at the age of 24 months. He did not speak but showed relatively good comprehension skills. He showed autistic-like features and autoaggression. An MRI scan of the brain at the age of 3 years 2 months showed mild periventricular gliosis. EEG and hearing test were normal. Ophthalmological examination showed hypermetropia.

\section{Cytogenetic analysis}

Standard cytogenetic analysis ${ }^{8}$ was performed on cultured lymphocytes with G-banding techniques at a resolution of 450-500 bands per haploid genome. Fluorescence in situ hybridization with probe TelVysion 9q SpectrumOrange (Abbott Laboratories, Abbott Park, IL, USA) was performed.

\section{Array CGH analysis}

CGH analysis was performed on the $400 \mathrm{~K}$ Oligonucleotide Array (Agilent Technologies, Santa Clara, CA, USA), according to the manufacturer's instructions. Data were analyzed using the CGHPRO software (Ullmann, Berlin, Germany).

${ }^{1}$ Institut für Klinische Genetik, Medizinische Fakultät Carl Gustav Carus, Technische Universität Dresden, Dresden, Germany; ${ }^{2}$ Institut für Humangenetik, Universitätsklinikum Tübingen, Tübingen, Germany; ${ }^{3}$ Max Planck Institut für Molekulare Genetik, Berlin, Germany; ${ }^{4}$ Institut für Humangenetik, Universitätsklinikum Leipzig, Leipzig, Germany *Correspondence: Dr D Mitter, Institute of Human Genetics, University Hospital Leipzig, Philipp-Rosenthal-Str. 55, Leipzig D-04103, Germany. Tel: + 49 3419723825; Fax: + 49 3419723819; E-mail: diana.mitter@medizin.uni-leipzig.de

Received 16 August 2012; revised 26 October 2012; accepted 1 November 2012; published online 12 December 2012 

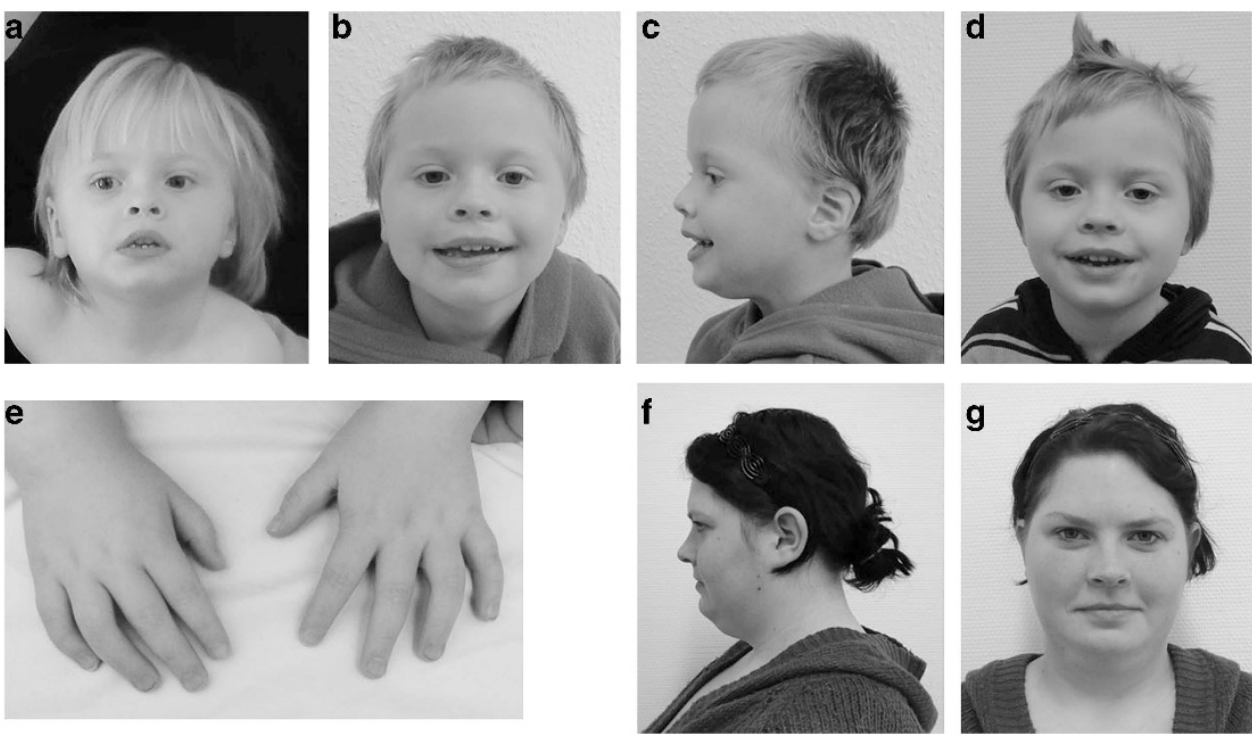

Figure 1 Facial phenotype. (a) Patient at the age of 3 years 1 month, (b, c) at 3 years 10 months, and (d) at 4 years 4 months. Facial dysmorphism includes anteverted nares, thin upper lip, everted lower lip, large mouth, and flat mid face. (e) Hands show tapering fingers. (f, $\mathbf{g}$ ) Mother of the patient at the age of 23 years with mild facial dysmorphism, including upslanting palpebral fissures, thin upper lip, and flat midface. The colour reproduction of this figure is available at the European Journal of Human Genetics online.

\section{Mutation analysis on genomic level}

Genomic DNA was derived from peripheral blood and oral mucosa. The 27 EHMT1 exons defined by RefSeq entry NM_024757.4, and flanking intronic regions were PCRamplified and sequenced using the BigDye-terminator chemistry (Applied Biosystems Deutschland GmbH, Darmstadt, Germany).

\section{Mutation analysis on cDNA level}

Total RNA from short-term cultivated lymphocytes was isolated with PAXgene Blood RNA Kit (Qiagen, Hilden, Germany). Using the SuperScript VILO cDNA synthesis kit (Invitrogen, Darmstadt, Germany), $1 \mu \mathrm{g}$ total RNA was transcribed to first-strand cDNA with random primers provided by the supplier. Amplification and sequencing of EHMT1 CDNA was conducted with primers specific for exons 15 (ex15F, CACCAGAATAAGCGCTCTC) and 20 (ex20R, GGCGTCTCTCCTTCCTTGTT). Obtained sequences were compared with reference sequence (UCSC, GRCh37/hg19 assembly).

\section{RESULTS}

Cytogenetic, molecular cytogenetic, and array CGH analysis Conventional cytogenetic analysis of the patient revealed a normal male karyotype. Fluorescence in situ hybridization analysis showed normal signals. Array CGH analysis revealed no potentially pathogenic copy number variations.

\section{Mutation analysis of EHMT1}

Sequencing of the exon-targeted EHMT1-amplicons revealed a novel G-to-A transition (NM_024757.4:c.2712+1G >A) abolishing the invariable consensus GT splice donor of intron 18 (Figure 2a) in the patient and his mother. Although the patient carries this mutation in a clearly heterozygous state, his mother shows mosaicism in peripheral blood (Figure 2b). Within her oral mucosa, the mutation was below detection level. The father and the younger brother of the patient did not carry the mutation. To determine the effect of the c. $2712+1 \mathrm{G}>$ A point mutation on splicing, total RNA of the patient and his parents was obtained from peripheral blood. A cDNA-portion of EMHT1, spanning exons 15-20, was amplified. Sequencing revealed three different transcripts (Figure 2c): besides the normal EHMT1 transcript produced by all family members (Figure 2c), mother and son uniquely express two additional aberrant transcripts. The first transcript results from the misapplication of a GTdinucleotide, which resides $4 \mathrm{bp}$ upstream of the original splice donor (Figure 2a). As a consequence, the resulting transcript contains four additional nucleotides between exons 18 and 19. The second aberrant transcript is the result of exon skipping of exon 18. This transcript produces a PCR-product that is $105 \mathrm{bp}$ shorter than the amplicon from normal cDNA (data not shown). According to Bioanalyzer quantification, the exon $17 / 19$ fusion represented $13.6 \%$ of the total PCR product obtained from the patient's cDNA and $10.1 \%$ of the total PCR product obtained from the maternal cDNA. To separate the 'exon $18+4$ bp'-PCR-product, we sequenced the RT-PCR product mixture and compared the peak heights of the 'exon $18+4$ bp'-PCRproduct with the peaks that are attributed to the normal transcript. In the patient, the aberrant 'exon $18+4 \mathrm{bp}$ '-PCR product constitutes approximately $20 \%$ of the total RT-PCR product, in the mother roughly $10 \%$.

\section{DISCUSSION}

We report on a patient with Kleefstra syndrome and a novel EHMT1 splice-site mutation. The c. $2712+1 \mathrm{G}>\mathrm{A}$ mutation has at least two different consequences at mRNA level. We detected two different splice variants. One variant contains $4 \mathrm{bp}$ of intronic sequence and is very likely to create a non-functional truncated EHMT1 protein. The other variant is more interesting: as skipping of exon 18 does not disrupt the reading frame, the resulting mRNA theoretically has the potential to produce a near-full length EHMT1 protein with just 35 amino acids missing (p.(Asp870_Asn904del)). This protein variant would lack ANK5, the fifth of eight consecutive ankyrin repeats in EHMT1. ${ }^{9}$ Given the fact that the patient displays the characteristic clinical features of Kleefstra syndrome, we have to conclude that the near-full length EHMT1 protein variant, if produced at all, is either not stable in the cells or not capable to attenuate pathogenicity.

The detection of mosaicism for a loss-of-function mutation in EHMT1 in the healthy mother indicates that Kleefstra syndrome is 


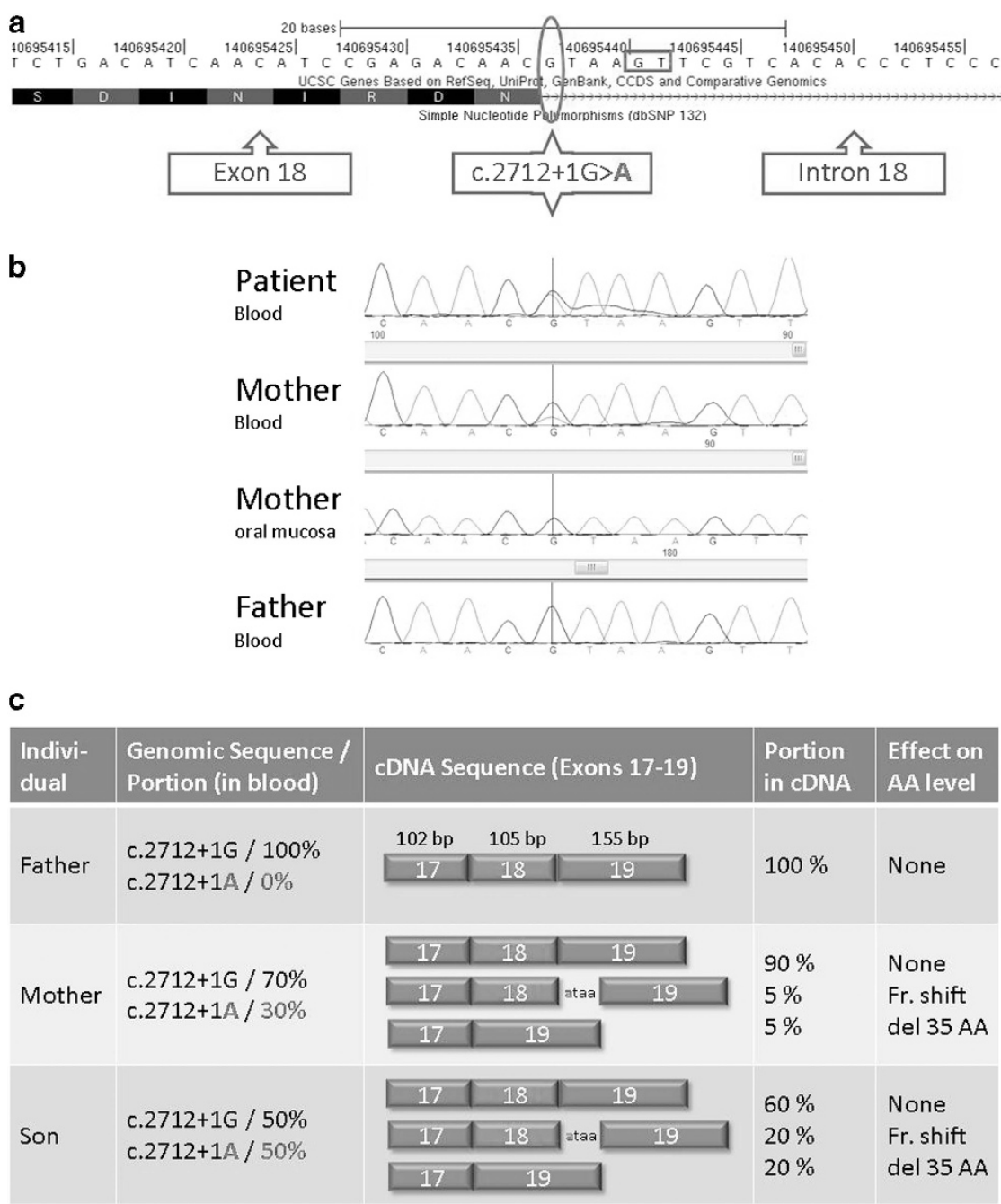

Figure 2 (a) Genomic position, (b) familial segregation and (c) transcriptional consequences of the EHMT1 mutation c.2712+1G>A. The red ellipse circles the mutation site in the UCSC genome browser (hg19 assembly); the red box frames the GT-dinucleotide used as splice-donor surrogate in the mutated EHMT1 allele. The affected son is heterozygous ( $\mathbf{b}$, row 1), his mother shows mosaicism in peripheral blood (b, row 2 ) and lacks the mutation in her oral mucosa. Both express two different types of aberrant transcripts (c) in different amounts (c, rows 2 and 3 ), whereas the unaffected father (b, row 4) only expresses the normal transcript (c, row 1). The colour reproduction of this figure is available at the European Journal of Human Genetics online.

not created unless a high degree of mosaicism is reached and/or certain tissues are affected. This is in line with observations made for a mosaic carrier of the $9 \mathrm{q} 34.3$ deletion, where $80 \%$ of the cells in a buccal swab sample carried a partial deletion of the EHMT1 gene. ${ }^{4}$ As the clinical features of Kleefstra syndrome involve tissues developing from different germ layers, a non-inherited (i.e. post-zygotic) Kleefstra syndrome causing mutation probably needs to occur in the very early stages of development in a cell that contributes to both germline and somatic tissues. Unfortunately, there is no way to infer the degree of germline mosaicism from the extent of somatic mosaicism within an individual. But as shown recently for two siblings with Pitt-Hopkins syndrome, ${ }^{10}$ a high degree of somatic mosaicism in a carrier bears the risk of familial recurrence in the offspring. Therefore, parents of a child with Kleefstra syndrome should always be checked for mosaicism for the respective mutation. In cases, where parental mosaicism is observed, the increased risk of familial recurrence has to be addressed within the genetic counseling of those families.

\section{CONFLICT OF INTEREST}

The authors declare no conflict of interest.

\section{ACKNOWLEDGEMENTS}

We thank the patient and his family for their cooperation and the cooperating physicians for referral of the patient, especially Lothar Bergmann

(Universitätskinderklinik Leipzig). We thank Professor Eberhard Passarge and the Executive Board of the University of Leipzig Hospitals and Clinics for continuous support. Array CGH analysis was performed at the Max-PlanckInstitute of Molecular Genetics Berlin, member of the 'German Mental Retardation Network' (MRNET), funded by the German Federal Ministry of Education and Research (BMBF) as part of the National Genome Research Network (NGFNplus/www.ngfn.de/englisch/15.htm, project reference numbers 01GS08164, 01GS08167).

\footnotetext{
1 Kleefstra T, Smidt M, Banning MJ et al: Disruption of the gene euchromatin histone methyl transferase1 (Eu-HMTase1) is associated with the 9q34 subtelomeric deletion syndrome. J Med Genet 2005; 42: 299-306.
} 
2 Kleefstra T, Brunner HG, Amiel J et al: Loss-of-function mutations in euchromatin histone methyl transferase 1 (EHMT1) cause the 9q34 subtelomeric deletion syndrome. Am J Hum Genet 2006; 79: 370-377.

3 Willemsen $\mathrm{MH}$, Vulto-van Silfhout AT, Nillesen WM et al: Update on Kleefstra Syndrome. Mol Syndromol 2012; 2: 202-212.

4 Willemsen MH, Beunders G, Callaghan M et al: Familial Kleefstra syndrome due to materna somatic mosaicism for interstitial 9q34.3 microdeletions. Clin Genet 2011; 80: 31-38.

5 Yatsenko SA, Brundage EK, Roney EK, Cheung SW, Chinault AC, Lupski JR: Molecular mechanisms for subtelomeric rearrangements associated with the 9q34.3 microdeletion syndrome. Hum Mol Genet 2009; 18: 1924-1936.

6 Kleefstra T, van Zelst-Stams WA, Nillesen WM et al: Further clinical and molecular delineation of the $9 q$ subtelomeric deletion syndrome supports a major contribution of EHMT1 haploinsufficiency to the core phenotype. J Med Genet 2009; 46: 598-606.

7 Kleefstra T, Nillesen WM, Yntema HG: Kleefstra syndrome; in Pagon RA, Bird TD, Dolan CR, Stephens K, Adam MP (eds) GeneReviews. Seattle, WA: University of Washington, Seattle, 1993 2010/10/15 (edn).

8 Seabright M: Human chromosome banding. Lancet 1972; 1: 967.

9 Collins RE, Northrop JP, Horton JR et al: The ankyrin repeats of G9a and GLP histone methyltransferases are mono- and dimethyllysine binding modules. Nat Struct Mol Biol 2008; 15: 245-250.

10 Steinbusch C, van Roozendaal K, Tserpelis D et al: Somatic mosaicism in a mother of two children with Pitt-Hopkins syndrome. Clin Genet 2012 